\title{
Projective synchronization of nonidentical fractional-order neural networks based on sliding mode controller
}

\author{
Zhixia Ding ${ }^{\mathrm{a}, \mathrm{b}}$, Yi Shen ${ }^{\mathrm{a}, \mathrm{b}, *}$ \\ ${ }^{a}$ School of Automation, Huazhong University of Science and Technology, Wuhan 430074, China \\ ${ }^{b}$ Key Laboratory of Image Processing and Intelligent Control of Education Ministry of China, Wuhan 430074, China
}

\begin{abstract}
This paper investigates global projective synchronization of nonidentical fractional-order neural networks (FNNs) based on sliding mode control technique. We firstly construct a fractional-order integral sliding surface. Then, according to the sliding mode control theory, we design a sliding mode controller to guarantee the occurrence of the sliding motion. Based on fractional Lyapunov direct methods, system trajectories are driven to the proposed sliding surface and remain on it evermore, and some novel criteria are obtained to realize global projective synchronization of nonidentical FNNs. As the special cases, some sufficient conditions are given to ensure projective synchronization of identical FNNs, complete synchronization of nonidentical FNNs and anti-synchronization of nonidentical FNNs. Finally, one numerical example is given to demonstrate the effectiveness of the obtained results.
\end{abstract}

Keywords: Projective synchronization; fractional-order neural networks; sliding mode controller; fractional-order integral sliding surface

\section{Introduction}

Fractional calculus, which is a generalization of integer-order integration and differentiation to its non-integer counter part, has been intensively investigated in many fields, covering dynamics of complex materials or porous media (Carpinteri \& Cornetti et al., 2004), fluid mechanics (Tripathi \& Pandey et al., 2010), bioengineering (Magin, 2004, 2010; Magin \& Ovadia, 2008), viscoelasticity (Soczkiewicz, 2002), etc. As fractional calculus has infinite memory, and has proven to be an excellent tool for the description of memory and hereditary properties of various materials and processes (Chen \& Ye et al., 2010; Isfer \& Lenzi et al., 2010; Kilbas \& Marzan, 2005; Szabo \& Wu, 2000), it is easy to see that the incorporation of a memory term into a neural network model is an extremely important improvement. In recent years, fractional-order neural networks (FNNs) have attracted attentions of many researchers, and various dynamical behaviors of FNNs have been widely investigated, such as Chen \& Chai et al. (2013); Kaslik \& Sivasundaram (2012); Wang \& Yu et al. (2013); Yu \& Hu et al. (2012); Zhou \& Li et al. (2008); Zou \& Qu et al. (2014).

Since Pecora and Carroll (Pecora \& Carroll, 1990) firstly put forward chaos synchronization in 1990, more and more researchers pay enough attentions to studying synchronization. The increasing interest in researching synchronization stems from its potential applications in cryptography, secure communication, optimization of nonlinear systems performance, modeling brain activity, and chemical reaction (Boccaletti \& Kurths et al., 2002; Chen \& Dong, 1998; Ojalvo \& Roy, 2001; Ott

\footnotetext{
${ }^{*}$ Corresponding author.

Email address: yishen64@163.com (Yi Shen)
}

\& Grebogi et al., 1990; Sprott, 2003; Yang \& Chua, 1997). So far, various types of fractional-order synchronization results emerge in large numbers, such as complete synchronization (Ding et al., 2016; Yan \& Li, 2007), generalized synchronization (Wu \& Lai et al., 2012), phase synchronization (Erjaee \& Momani, 2008), lag synchronization (Zhu \& He et al., 2011), etc. As pointed out in Wang \& He (2008), projective synchronization, amongst all kinds of synchronization, can obtain faster communication for its proportional feature in application to secure communications. Subsequently, a serious of results about projective synchronization appear, such as Bao \& Cao (2015); Hu \& Xu et al. (2008); Si \& Sun et al. (2012); Xin \& Chen et al. (2011); Yu \& Hu et al. (2014).

However, to the best of our knowledge, most reports are concerned with the projective synchronization problem for identical fractional-order systems. In practice, due to the mismatched parameters and functions which are unavoidable in real implementation, the drive system and response system are not identical. From the point of view of engineering, it is very difficult to keep the two systems to be identical all the time (Huang \& Feng, 2009). Therefore, it is significant to study projective synchronization problem of nonidentical FNNs.

To deal with synchronization of FNNs, there exist different control approaches, such as linear feedback control (Chen \& Zeng et al., 2014; Zhang \& Yu et al., 2015), adaptive control (Bao \& Cao, 2015; Yu \& Hu et al., 2014), and so on. Sliding mode control, as a robust variable structure control method, is an important control technique. Its advantages include fast response, low sensitivity to external noises, robustness to the system uncertainties and easy realization. The main idea of sliding mode control is forcing the system state trajectories to some predefined sliding surfaces by using a discontinuous con- 
troller, and the system on sliding surfaces has desired properties such as stability. In addition, sliding mode controller include an equivalent control part that describes the behaviors of the system when the trajectories stay over the sliding surface, and a variable structure control part that enforces the trajectories to reach the sliding surface and remain on it evermore.

Motivated by the above discussions, in this paper, we focus our attentions on the global projective synchronization of nonidentical FNNs in the sense of Caputo fractional derivation. Firstly, by considering the measured output of system, a fractional-order integral sliding surface is properly constructed. To guarantee the existence of the sliding motion, we design a sliding mode controller by using sliding mode control theory. Then, based on fractional Lyapunov direct methods and the properties of Caputo fractional-order derivative, reachability of the specified sliding surface is analyzed, and some sufficient criteria for the global projective synchronization of nonidentical FNNs are presented. Besides, the global projective synchronization of identical FNNs are proved. Finally, by selecting different projection coefficient, the obtained results can be used to achieve globally asymptotically complete synchronization and globally asymptotically anti-synchronization of nonidentical FNNs.

The organization of this paper is as follows. The system and some preliminaries are introduced in Section 2. In Section 3, sufficient criteria are established based on a fractional-order integral sliding mode controller, sliding mode control theory and fractional Lyapunov direct methods. Then, numerical simulations are given to demonstrate the effectiveness of the obtained results in Section 4. Finally, conclusions are drawn in Section 5.

\section{Preliminaries}

Notations: Through this paper, $\mathbb{R}$ is the space of real number, $\mathbb{N}_{+}$is the set of positive integers, $\mathbb{C}$ is the space of complex number, $\mathbb{R}^{n}$ denotes the $n$-dimensional Euclidean space, and $\mathbb{R}^{m \times n}$ denotes the set of all $m \times n$ real matrices. $\operatorname{sgn}(\cdot)$ is symbolic function. $[\cdot, \cdot]$ represents the interval and $\operatorname{diag}\{\cdots\}$ denotes a block-diagonal matrix. If not stated explicitly, matrices are assumed to have compatible dimensions for algebraic operations. In addition, $C^{r}\left(\left[t_{0},+\infty\right), \mathbb{R}\right)$ denotes the space consisting of r-order continuous differentiable functions from $\left[t_{0},+\infty\right)$ into $\mathbb{R}$.

In order to investigate FNNs, we firstly recall some definitions about fractional calculation and introduce some useful lemmas in this section.

\subsection{Caputo fractional-order derivative}

Definition 1 (Kilbas \& Srivastava et al., 2006; Podlubny, 1999). The fractional-order integral of order $\alpha$ for an integrable function $f(t):\left[t_{0},+\infty\right) \rightarrow \mathbb{R}$ is defined as

$$
{ }_{t_{0}} I_{t}^{\alpha} f(t)=\frac{1}{\Gamma(\alpha)} \int_{t_{0}}^{t}(t-\tau)^{\alpha-1} f(\tau) d \tau
$$

where $\alpha>0$, and $\Gamma(\cdot)$ is the Gamma function which is defined by

$$
\Gamma(z)=\int_{0}^{\infty} e^{-t} t^{z-1} d t,(\operatorname{Re}(z)>0),
$$

where $\operatorname{Re}(z)$ is the real part of $z$.

Definition 2 (Kilbas \& Srivastava et al., 2006; Podlubny, 1999). The Caputo fractional-order derivative of order $\alpha$ for a function $f(t) \in C^{n+1}\left(\left[t_{0},+\infty\right), \mathbb{R}\right)$ is defined as

$$
{ }_{t_{0}} D_{t}^{\alpha} f(t)=\frac{1}{\Gamma(n-\alpha)} \int_{t_{0}}^{t} \frac{f^{(n)}(\tau)}{(t-\tau)^{\alpha-n+1}} d \tau,
$$

where $t \geq t_{0}$ and $n$ is a positive integer such that $n-1<\alpha<n$. Particularly, when $0<\alpha<1$,

$$
{ }_{t_{0}} D_{t}^{\alpha} f(t)=\frac{1}{\Gamma(1-\alpha)} \int_{t_{0}}^{t} \frac{f^{\prime}(\tau)}{(t-\tau)^{\alpha}} d \tau
$$

Several important properties about Caputo fractional-order derivative are listed below (Aghababa, 2013a; Li \& Deng, 2007; Podlubny, 1999).

Property 1. ${ }_{t 0} D_{t}^{\alpha} c=0$ holds, where $c$ is any constant.

Property 2. For any constants $v_{1}$ and $v_{2}$, the linearly of Caputo fractional-order derivative is described by

$$
{ }_{t_{0}} D_{t}^{\alpha}\left(v_{1} f(t)+v_{2} g(t)\right)=v_{1 t_{0}} D_{t}^{\alpha} f(t)+v_{2} t_{0} D_{t}^{\alpha} g(t) .
$$

Property 3. ${ }_{t_{0}} D_{t t_{0}}^{\alpha} I_{t}^{\beta} f(t)={ }_{t_{0}} D_{t}^{\alpha-\beta} f(t)$ where $\alpha \geq \beta \geq 0$. Especially, when $\alpha=\beta, t_{0} D_{t t_{0}}^{\alpha} I_{t}^{\alpha} f(t)=f(t)$.

Property 4. The Leibniz's rule for fractional differentiation is given as:

$$
{ }_{t_{0}} D_{t}^{\alpha}(\phi(t) f(t))=\sum_{k=0}^{\infty} \frac{\Gamma(1+\alpha)}{\Gamma(1+k) \Gamma(\alpha-k+1)} \phi^{(k)}(t){ }_{t_{0}} D_{t}^{\alpha-k} f(t),
$$

if $\phi(t)$ and $f(t)$ and all their derivatives are continuous in the interval $\left[t_{0}, t\right]$, where $\phi^{(k)}(t)$ is the integer-order derivative of order $k$ for function $\phi(t)$ and ${ }_{t_{0}} D_{t}^{\alpha-k} f(t)$ is Caputo fractional-order derivative of order $\alpha-k$ for function $f(t)$.

Property 5. If $x(t) \in C^{1}[0, T]$ for some $T>0$, then

$$
{ }_{0} D_{t}^{\alpha_{1}}{ }_{0} D_{t}^{\alpha_{2}} x(t)={ }_{0} D_{t}^{\alpha_{2}}{ }_{0} D_{t}^{\alpha_{1}} x(t)={ }_{0} D_{t}^{\alpha_{1}+\alpha_{2}} x(t), \quad t \in[0, T],
$$

where $\alpha_{1}>0, \alpha_{2}>0$ and $\alpha_{1}+\alpha_{2} \leq 1$.

In addition, some necessary Lemmas about Caputo fractional-order derivative are given.

Lemma 1 (Kilbas \& Srivastava et al., 2006). Let $\Omega=[a, b]$ be an interval on the real axis $\mathbb{R}$, let $n=[\alpha]+1$ for $\alpha \notin \mathbb{N}_{+}$or $n=\alpha$ for $\alpha \in \mathbb{N}_{+}$. If $x(t) \in C^{n}[a, b]$, then

$$
{ }_{a} I_{t}^{\alpha}{ }_{a} D_{t}^{\alpha} x(t)=x(t)-\sum_{k=0}^{n-1} \frac{x^{(k)}(a)}{k !}(t-a)^{k}, n-1<\alpha \leq n,
$$

where ${ }_{a} I_{t}^{\alpha}$ is fractional-order integral of order $\alpha$ and ${ }_{a} D_{t}^{\alpha}$ is Caputo fractional-order derivative of order $\alpha$. In particular, if $0<\alpha \leq 1$ and $x(t) \in C^{1}[a, b]$, then

$$
{ }_{a} I_{t a}^{\alpha} D_{t}^{\alpha} x(t)=x(t)-x(a) .
$$


Lemma 2 (Zhang \& Yu et al., 2015). If $h(t) \in C^{1}([0,+\infty], \mathbb{R})$ denotes a continuously differentiable function, the following inequality holds almost everywhere.

$$
{ }_{0} D_{t}^{\alpha}|h(t)| \leq \operatorname{sgn}(h(t))_{0} D_{t}^{\alpha} h(t), \quad 0<\alpha<1 .
$$

\subsection{Mittag-Leffler type function and Mittag-Leffler stability}

Similar to the exponential function frequently used in the solutions of integer-order systems, a function frequently used in the solutions of fractional-order systems is the Mittag-Leffler function. The Mittag-Leffler function with two parameters appear most frequently and have the following form:

$$
E_{\alpha, \beta}(z)=\sum_{k=0}^{\infty} \frac{z^{k}}{\Gamma(\alpha k+\beta)},
$$

where $\alpha>0, \beta>0$ and $z \in \mathbb{C}$. For $\beta=1$, we have $E_{\alpha}(z)=$ $E_{\alpha, 1}(z)$ defined as

$$
E_{\alpha}(z)=\sum_{k=0}^{\infty} \frac{z^{k}}{\Gamma(\alpha k+1)},
$$

where $\alpha>0$ and $z \in \mathbb{C}$. Especially, $E_{1,1}(z)=e^{z}$, where $\alpha=\beta=$ 1.

And the Laplace transform of two-parameter Mittag-Leffler function is

$$
\mathscr{L}\left\{t^{\beta-1} E_{\alpha, \beta}\left(-\lambda t^{\alpha}\right)\right\}=\frac{s^{\alpha-\beta}}{s^{\alpha}+\lambda},\left(\operatorname{Re}(s)>|\lambda|^{\frac{1}{\alpha}}\right),
$$

where $t \geq 0, s$ is the variable in Laplace domain and $\operatorname{Re}(s)$ is the real part of $s, \lambda \in \mathbb{R}$.

Consider a $\mathrm{n}$-dimensional fractional-order system

$$
\left\{\begin{array}{l}
{ }_{0} D_{t}^{\alpha} x(t)=f(t, x) \\
x\left(t_{0}\right)=x_{t_{0}}
\end{array}\right.
$$

where $\alpha \in(0,1), x=\left(x_{1}, x_{2}, \ldots, x_{n}\right)^{T} \in \mathbb{R}^{n}, t_{0} \geq 0$ is the initial time and $f:\left[t_{0},+\infty\right) \times \mathbb{R}^{n} \rightarrow \mathbb{R}^{n}$ is piecewise continuous on $t$ and satisfies locally Lipschitz condition on $x$. In what follows, we give the definition of Mittag-Leffler stability and fractionalorder Lyapunov direct methods for system (1).

Definition 3 (Li et al., 2010). Let $x=0$ be an equilibrium point of system (1), the solution of system (1) is said to be MittagLeffler stable if

$$
\|x(t)\| \leq\left\{m\left[x\left(t_{0}\right)\right] E_{\alpha}\left(-\lambda\left(t-t_{0}\right)^{\alpha}\right)\right\}^{b},
$$

where $\lambda>0, b>0, m(0)=0, m(x) \geq 0, m(x)$ satisfies locally Lipschitz condition on $x \in \mathbb{R}^{n}$ with Lipschitz constant $m_{0}$, and $\|\cdot\|$ denotes an arbitrary norm.

As asymptotic stability of the systems can be obtained by using the Lyapunov direct method. Next, we will give the Lyapunov direct methods of fractional-order systems, which lead to the Mittag-Leffler stability.

Lemma 3 (Li et al., 2009, 2010). For $t_{0}=0$, and let $x=0$ be an equilibrium point for the fractional-order system (1). If there exists $V(t, x(t)):[0,+\infty) \times \mathbb{D} \rightarrow \mathbb{R}^{n}$ be a continuously differentiable function and locally Lipschitz with respect to $x$ such that

$$
\alpha_{1}\|x\|^{a} \leq V(t, x(t)) \leq \alpha_{2}\|x\|^{a b}
$$

$$
{ }_{0} D_{t}^{\beta} V(t, x(t)) \leq-\alpha_{3}\|x\|^{a b},
$$

where $\mathbb{D} \subset \mathbb{R}^{n}$ be a domain containing the origin, $t \geq 0, x \in \mathbb{D}$, $\beta \in(0,1), \alpha_{1}, \alpha_{2}, \alpha_{3}, a$ and $b$ are arbitrary positive constants. Then $x=0$ is Mittag-Leffler stable. If the assumptions hold globally on $\mathbb{R}^{n}$, then $x=0$ is globally Mittag-Leffler stable.

Remark 1. Mittag-Leffler stability implies asymptotic stability. Next, the fractional Lyapunov direct method is described by the class- $\mathcal{K}$ functions.

Lemma 4 (Li et al., 2009, 2010). For $t_{0}=0$, and let $x=0$ be an equilibrium point for the fractional-order system (1). If there exists a Lyapunov function $V(t, x(t)):[0,+\infty) \times \mathbb{D} \rightarrow \mathbb{R}^{n}$ and class- $\mathcal{K}$ functions $\alpha_{i}(i=1,2,3)$ satisfying

$$
\alpha_{1}(\|x\|) \leq V(t, x(t)) \leq \alpha_{2}(\|x\|),
$$

$$
{ }_{0} D_{t}^{\beta} V(t, x(t)) \leq-\alpha{ }_{3}(\|x\|),
$$

where $\beta \in(0,1)$ and $\mathbb{D} \subset \mathbb{R}^{n}$ be a domain containing the origin. Then the equilibrium point of the system (1) is asymptotically stable.

\subsection{System description}

In this paper, we consider a class of FNNs as the drive system described by

$$
{ }_{0} D_{t}^{\alpha} x_{i}(t)=-c_{i} x_{i}(t)+\sum_{j=1}^{n} a_{i j} f_{j}\left(x_{j}(t)\right)+I_{i},
$$

or

$$
{ }_{0} D_{t}^{\alpha} x(t)=-C x(t)+A f(x(t))+I,
$$

where ${ }_{0} D_{t}^{\alpha}$ denotes the Caputo fractional derivative of order $\alpha$ and $0<\alpha<1 ; x(t)=\left(x_{1}(t), x_{2}(t), \ldots, x_{n}(t)\right)^{T} \in \mathbb{R}^{n}$ and $x_{i}(t)$ corresponds to the state of the $i$ th unit at time $t . C=$ $\operatorname{diag}\left(c_{1}, c_{2}, \ldots, c_{n}\right)$ is a diagonal self connection weight matrix with $c_{i}>0, i=1,2, \ldots, n ; A=\left(a_{i j}\right)_{n \times n}$ is the interconnection weight matrix; $f(x)=\left(f_{1}\left(x_{1}\right), f_{2}\left(x_{2}\right), \ldots, f_{n}\left(x_{n}\right)\right)^{T}: \mathbb{R}^{n} \rightarrow \mathbb{R}^{n}$ is a diagonal mapping where $f_{j}\left(x_{j}\right)$ denotes the activation function of the $j$ th neuron, and $I=\left[I_{1}, I_{2}, \ldots, I_{n}\right]^{T}$ is the external input vector. It is assumed that the measured output of system (7) is dependent on the instantaneous state with the following form:

$$
y(t)=P x(t),
$$

where $y(t) \in \mathbb{R}^{m}$, and $P \in \mathbb{R}^{m \times n}$ is known constant matrix.

The corresponding response system is given by

$$
{ }_{0} D_{t}^{\alpha} z(t)=-D z(t)+B g(z(t))+H+u(t),
$$


where $z(t) \in \mathbb{R}^{n}$ is the state vector of the response system, and $u(t)$ is the control input to be designed.

Throughout this paper, the activation functions $f(\cdot)$ and $g(\cdot)$ satisfy the following assumption:

Assumption 1: For all $j=1,2, \ldots, n$, there exist constants $l_{j}^{-}, l_{j}^{+}, m_{j}^{-}$and $m_{j}^{+}$such that for any $u \neq v \in \mathbb{R}$, the following conditions are always satisfied:

$$
l_{j}^{-} \leq \frac{f_{j}(u)-f_{j}(v)}{u-v} \leq l_{j}^{+}
$$

and

$$
m_{j}^{-} \leq \frac{g_{j}(u)-g_{j}(v)}{u-v} \leq m_{j}^{+}
$$

Remark 2. Obviously, the constants $l_{j}^{-}, l_{j}^{+}, m_{j}^{-}, m_{j}^{+}$in assumption 1 are allowed to be positive, negative or zero, and the activation functions $f(\cdot)$ and $g(\cdot)$ are neither bounded nor monotonous nondecreasing. Compared with the previous assumptions: monotonous nondecreasing function (Gan \& Xu et al., 2011; Huang \& Feng, 2009), bounded function (Wang et al., 2015a,b) and common Lipschitz-continuous function (Bao \& Cao, 2015; Chen \& Zeng et al., 2014; Yu \& Hu et al., 2014; Zhang \& Yu et al., 2015), our assumption is weaker. Which makes our results admit less conservation.

It is our intention in this paper to investigate the projective synchronization of drive system (7) and response system (9). We give the definition of projective synchronization as follows.

Definition 4 ( $\mathrm{Yu} \& \mathrm{Hu}$ et al., 2014). If there exists nonzero constant $\beta \in \mathbb{R}$, such that for any two solutions $x(t)$ and $z(t)$ of drive system (7) and response system (9) with different initial values denoted by $x_{0}$ and $z_{0}$, one can get

$$
\lim _{t \rightarrow+\infty}\|z(t)-\beta x(t)\|=0
$$

then, the drive system (7) and response system (9) can achieve globally asymptotically projective synchronization, where $\|\cdot\|$ denotes the Euclidean norm, and $\beta$ denotes the projective coefficient.

Remark 3. If $\beta=1$, the system (7) and (9) can achieve globally asymptotically complete synchronization; if $\beta=-1$, the system (7) and (9) can achieve globally asymptotically antisynchronization. If $\beta=0$, the above inequality in the definition is derived into the following form

$$
\lim _{t \rightarrow+\infty}\|z(t)\|=0
$$

that is the (9) is globally asymptotically stabilized to the origin.

\section{Main results}

Let $e(t)=z(t)-\beta x(t)$ be the synchronization error, from system (7) and system (9), the error system is

$$
\begin{aligned}
{ }_{0} D_{t}^{\alpha} e(t)= & -D e(t)+B \psi(e(t))+\beta(C-D) x(t)+B g(\beta x(t)) \\
& -\beta A f(x(t))+H-\beta I+u(t),
\end{aligned}
$$

where $\psi(e(t))=g(z(t))-g(\beta x(t)), C, A, I$ are matrices which are the same as system (7) and $D, B, H$ are matrices which are the same as system (9).

To study the projective synchronization of different FNNs (7) and (9), that is equivalent to study the asymptotic stability of the equilibrium point for error system (10). It is easy to see that parameters and functions between the drive and response systems are different, and the dynamic behaviors of the error system depend on the state $x(t)$ of the drive system (7), so the common output feedback controller can not be applied. Here, a fractional integral sliding mode control approach will be adopted to deal with the projective synchronization problem.

Sliding mode control, as a robust variable structure control method, generally consists of the following two main steps. First, construct an appropriate sliding surface with some desired properties. Second, develop a input signal $u(t)$ to force the system trajectory onto the sliding surface and subsequently stay on it evermore.

Considering the measured output $y(t)$ of system (7), we define the sliding surface as

$$
\begin{aligned}
S(t) & =e(t)+{ }_{0} I_{t}^{\alpha}[C e(t)-B \psi(e(t))-K(\beta y(t)-P z(t))] \\
& =e(t)+{ }_{0} I_{t}^{\alpha}[(C+K P) e(t)-B \psi(e(t))],
\end{aligned}
$$

where the gain matrix $K \in \mathbb{R}^{n \times m}$ is to be chosen suitably, $P$ is coefficient matrix in (8), $C$ and $B$ are defined in system (7) and (9), respectively.

For convenience, we need to get the equivalent sliding surface through a combination of error system (10) and Lemma 1, as described next.

From error system (10), one can integrate the system (10) from 0 to $t$

$$
\begin{aligned}
{ }_{0} I_{t}^{\alpha}{ }_{0} D_{t}^{\alpha} e(t)= & { }_{0} I_{t}^{\alpha}[-D e(t)+B \psi(e(t))+\beta(C-D) x(t)+B g(\beta x(t)) \\
& -\beta A f(x(t))+H-\beta I+u(t)]
\end{aligned}
$$

according to Lemma 1, we can obtain

$$
\begin{aligned}
e(t)= & e(0)+{ }_{0} I_{t}^{\alpha}[-D e(t)+B \psi(e(t))+\beta(C-D) x(t)+B g(\beta x(t)) \\
& -\beta A f(x(t))+H-\beta I+u(t)]
\end{aligned}
$$

then, from (11) and (12), we have

$$
\begin{aligned}
S(t)= & e(0)+{ }_{0} I_{t}^{\alpha}[(C-D+K P) e(t)+\beta(C-D) x(t) \\
& +B g(\beta x(t))-\beta A f(x(t))+H-\beta I+u(t)],
\end{aligned}
$$

which is the equivalent sliding surface.

According to the sliding mode control theory (Slotine \& $\mathrm{Li}$, 1991), when the fractional-order system (10) operates in sliding mode, the sliding surface and its derivative must satisfy

$$
S(t)=0 \text { and } \dot{S}(t)=0 .
$$

Based on property 5, we have $\dot{S}(t)={ }_{0} D_{t}^{1-\alpha}{ }_{0} D_{t}^{\alpha} S(t)$, then, $\dot{S}(t)=0$ implies ${ }_{0} D_{t}^{\alpha} S(t)=0$. From (13), property 1 and prop- 
erty 3 , one has

$$
\begin{aligned}
{ }_{0} D_{t}^{\alpha} S(t)= & { }_{0} D_{t}^{\alpha} e(0)+{ }_{0} D_{t 0}^{\alpha} I_{t}^{\alpha}[(C-D+K P) e(t)+\beta(C-D) x(t) \\
& +B g(\beta x(t))-\beta A f(x(t))+H-\beta I+u(t)] \\
= & (C-D+K P) e(t)+\beta(C-D) x(t)+B g(\beta x(t)) \\
& -\beta A f(x(t))+H-\beta I+u(t) \\
= & 0
\end{aligned}
$$

It thus follows from (14) that an equivalent control law can be designed as

$$
\begin{aligned}
u_{e q}(t)= & -(C-D+K P) e(t)-\beta(C-D) x(t)-B g(\beta x(t)) \\
& +\beta A f(x(t))-H+\beta I .
\end{aligned}
$$

Substituting (15) into (10), the sliding mode dynamics can be obtained and described by

$$
{ }_{0} D_{t}^{\alpha} e(t)=-(C+K P) e(t)+B \psi(e(t)),
$$

where $\psi(e(t))=g(z(t))-g(\beta x(t))$. It is easy to see that $e=0$ is an equilibrium point of system (16).

\subsection{Reachability analysis}

To drive system trajectories onto the sliding surface, a reaching law can be chosen as

$$
u_{r}=-k^{*}(\operatorname{sgn}(S(t)))
$$

where

$$
\operatorname{sgn}(S(t))=\left\{\begin{array}{cc}
-1, & S(t)<0 \\
0, & S(t)=0 \\
1, & S(t)>0
\end{array}\right.
$$

and $k^{*}$ is the switching gain, which is positive. Finally, the overall control $u(t)$ is determined by

$$
\begin{aligned}
u(t)= & u_{e q}+u_{r} \\
= & -(C-D+K P) e(t)-\beta(C-D) x(t)-B g(\beta x(t)) \\
& +\beta A f(x(t))-H+\beta I-k^{*}(\operatorname{sgn}(S(t))),
\end{aligned}
$$

To get the reachability analysis, we need to give the following assumption.

Assumption 2: Assume that the following inequality holds:

$$
\left|\sum_{k=1}^{\infty} \frac{\Gamma(1+\alpha)}{\Gamma(1+k) \Gamma(\alpha-k+1)} S^{(k)}(t)_{0} D_{t}^{\alpha-k} S(t)\right| \leq \eta\|S(t)\|,
$$

where $\alpha \in(0,1), \eta<k^{*}$ and $S(t)$ is the sliding surface defined in (11).

Theorem 1. Assume that the sliding surface is given by (11) and assumptions 2 holds, then the trajectories of the error system (10) based on the sliding mode control law (17) can be globally driven onto the sliding surface $S(t)=0$.

Proof. Constructing the following Lyapunov function:

$$
V(t)=S(t)^{2}
$$

basing on property 4 and assumption 2 , the fractional-order derivative the function (18) is given by

$$
\begin{aligned}
& { }_{0} D_{t}^{\alpha} V(t) \\
= & { }_{0} D_{t}^{\alpha}\left(S(t)^{2}\right) \\
= & S(t)_{0} D_{t}^{\alpha} S(t)+\sum_{k=1}^{\infty} \frac{\Gamma(1+\alpha)}{\Gamma(1+k) \Gamma(\alpha-k+1)} S^{(k)}(t)_{0} D_{t}^{\alpha-k} S(t) \\
\leq & S(t)_{0} D_{t}^{\alpha} S(t)+\eta\|S(t)\| \\
\leq & S(t)[(C-D+K P) e(t)+\beta(C-D) x(t)+B g(\beta x(t)) \\
& -\beta A f(x(t))+H-\beta I+u(t)]+\eta\|S(t)\| \\
= & S(t)\left[-k^{*}(\operatorname{sgn}(S(t)))\right]+\eta\|S(t)\| \\
= & -\left(k^{*}-\eta\right)\|S(t)\| .
\end{aligned}
$$

As $k^{*}-\eta>0$, the system states will converge to $S(t)=0$ asymptotically based on Lemma 4 . That is, the trajectory of the error system (10) is globally driven onto the specified sliding surface and maintained there for all subsequent time. This completes the proof.

\subsection{Stability analysis of the sliding motion}

In the following, the paper aims to find some sufficient stability criteria for the sliding motion (16). In order to obtain the main results, the following condition is needed in later study.

Assumption 3: For all $i, j=1, \ldots, n$, we have

$$
c_{i}-\sum_{j=1}^{n}\left(\sum_{l=1}^{m}\left|k_{j l} p_{l i}\right|\right)-\sum_{j=1}^{n}\left|b_{j i}\right| m_{i}>0
$$

where $c_{i}$ is the same constant as system (6), $p_{l j}$ is defined in (8), $b_{j i}$ is the coefficient of system (9), $m_{i}=\max \left\{\left|m_{i}^{-}\right|,\left|m_{i}^{+}\right|\right\}$where $m_{i}^{-}$and $m_{i}^{+}$are constant defined in the assumption 1 , and $k_{j l}$ is the gain in (11).

Theorem 2. Suppose that the assumptions 1 and 3 hold, the sliding mode dynamics (16) is globally asymptotically stable.

Proof. Firstly, the sliding mode dynamics (16) is equivalent to the following form

$$
{ }_{0} D_{t}^{\alpha} e_{i}(t)=-c_{i} e_{i}(t)-\sum_{j=1}^{n}\left(\sum_{l=1}^{m} k_{i l} p_{l j}\right) e_{j}(t)+\sum_{j=1}^{n} b_{i j} \psi_{j}\left(e_{j}(t)\right),
$$

where $\psi_{j}\left(e_{j}(t)\right)=g_{j}\left(z_{j}(t)\right)-g_{j}\left(\beta x_{j}(t)\right)$.

Choose the following positive definite function as a Lyapunov function candidate:

$$
V(t)=\|e(t)\|_{1}=\sum_{i=1}^{n}\left|e_{i}(t)\right|
$$

it is obvious that (21) satisfies (2) in Lemma 3.

Based on Lemma 2, we can obtain

$$
{ }_{0} D_{t}^{\alpha}\left|e_{i}(t)\right| \leq \operatorname{sgn}\left(e_{i}(t)\right)_{0} D_{t}^{\alpha} e_{i}(t) .
$$


Calculating the fractional-order derivatives of $V(t)$ along the solution of system (20) under (22),

$$
\begin{aligned}
{ }_{0} D_{t}^{\alpha} V(t)= & \sum_{i=1}^{n}{ }_{0} D_{t}^{\alpha}\left|e_{i}(t)\right| \\
\leq & \sum_{i=1}^{n} \operatorname{sgn}\left(e_{i}(t)\right)_{0} D_{t}^{\alpha} e_{i}(t) \\
= & \sum_{i=1}^{n} \operatorname{sgn}\left(e_{i}(t)\right)\left\{-c_{i} e_{i}(t)-\sum_{j=1}^{n}\left(\sum_{l=1}^{m} k_{i l} p_{l j}\right) e_{j}(t)\right. \\
& \left.+\sum_{j=1}^{n} b_{i j} \psi_{j}\left(e_{j}(t)\right)\right\} \\
= & -\sum_{i=1}^{n} \operatorname{sgn}\left(e_{i}(t)\right)\left(c_{i} e_{i}(t)\right) \\
& -\sum_{i=1}^{n} \operatorname{sgn}\left(e_{i}(t)\right)\left[\sum_{j=1}^{n}\left(\sum_{l=1}^{m} k_{i l} p_{l j}\right) e_{j}(t)\right] \\
& +\sum_{i=1}^{n} \operatorname{sgn}\left(e_{i}(t)\right)\left[\sum_{j=1}^{n} b_{i j} \psi_{j}\left(e_{j}(t)\right)\right] .
\end{aligned}
$$

From assumption 1 , one has $m_{j}^{-} \leq \frac{\psi_{j}\left(e_{j}(t)\right)}{e_{j}(t)} \leq m_{j}^{+}$, we denote $m_{j}=\max \left\{\left|m_{j}^{-}\right|,\left|m_{j}^{+}\right|\right\}$for $j=1,2, \ldots, n$ such that

$$
\left|\psi_{j}\left(e_{j}(t)\right)\right| \leq m_{j}\left|e_{j}(t)\right|
$$

Based on (24), the inequality (23) can be converted to the following form

$$
\begin{aligned}
{ }_{0} D_{t}^{\alpha} V(t) \leq & -\sum_{i=1}^{n} c_{i}\left|e_{i}(t)\right|+\sum_{i=1}^{n}\left[\sum_{j=1}^{n}\left(\sum_{l=1}^{m}\left|k_{i l} p_{l j}\right|\right)\left|e_{j}(t)\right|\right] \\
& +\sum_{i=1}^{n} \sum_{j=1}^{n}\left|b_{i j}\right| m_{j}\left|e_{j}(t)\right| \\
= & -\sum_{i=1}^{n} c_{i}\left|e_{i}(t)\right|+\sum_{i=1}^{n}\left[\sum_{j=1}^{n}\left(\sum_{l=1}^{m}\left|k_{j l} p_{l i}\right|\right)\right]\left|e_{i}(t)\right| \\
& +\sum_{i=1}^{n}\left[\sum_{j=1}^{n}\left|b_{j i}\right| m_{i}\right]\left|e_{i}(t)\right| \\
= & -\sum_{i=1}^{n}\left[c_{i}-\sum_{j=1}^{n}\left(\sum_{l=1}^{m}\left|k_{j l} p_{l i}\right|\right)-\sum_{j=1}^{n}\left|b_{j i}\right| m_{i}\right]\left|e_{i}(t)\right| .
\end{aligned}
$$

Let $\lambda_{i}=c_{i}-\sum_{j=1}^{n}\left(\sum_{l=1}^{m}\left|k_{j l} p_{l i}\right|\right)-\sum_{j=1}^{n}\left|b_{j i}\right| m_{i}$, and know $\lambda_{i}>0$ from assumption 3 , so we denote

$$
\lambda=\min _{1 \leq i \leq n} \lambda_{i}=\min _{1 \leq i \leq n}\left\{c_{i}-\sum_{j=1}^{n}\left(\sum_{l=1}^{m}\left|k_{j l} p_{l i}\right|\right)-\sum_{j=1}^{n}\left|b_{j i}\right| m_{i}\right\}>0,
$$

then, the inequality (25) can be replaced by

$$
{ }_{0} D_{t}^{\alpha} V(t) \leq-\lambda V(t)=-\lambda\|e(t)\|_{1}, \quad t \geq 0 .
$$

which implies (3) holds in Lemma 3. Based on Lemma 3, the equilibrium point $x=0$ is globally Mittag-Leffler stable. It means that the sliding mode dynamics (16) is globally asymptotically stable, i.e., $\|e(t)\| \rightarrow 0 \quad(t \rightarrow+\infty)$. The proof is completed.

Remark 4. As $e(t)=z(t)-\beta x(t)$, we can get directly from Theorem 2

$$
\|z(t)-\beta x(t)\| \rightarrow 0 \quad(t \rightarrow+\infty),
$$

that is, systems (7) and (9) are globally projective synchronized based on the controller (17).

When $C=D, A=B, I=H$ and $f=g$, the form and parameters of response system are the same as the drive system. The response system is

$$
{ }_{0} D_{t}^{\alpha} z(t)=-C z(t)+A f(z(t))+I+u(t) .
$$

We consider the projective synchronization between two identical FNNs. The sliding surface is described as follows

$$
\begin{aligned}
S(t) & =e(t)+{ }_{0} I_{t}^{\alpha}[C e(t)-A \psi(e(t))-K(\beta y(t)-P z(t))] \\
& =e(t)+{ }_{0} I_{t}^{\alpha}[(C+K P) e(t)-A \psi(e(t))]
\end{aligned}
$$

where $\psi(e(t))=f(z(t))-f(\beta x(t))$ and the gain matrix $K \in \mathbb{R}^{n \times m}$ is to be chosen suitably. The controller is designed by

$$
\begin{aligned}
u(t)= & u_{e q}+u_{r} \\
= & -K P e(t)-A f(\beta x(t))+\beta A f(x(t)) \\
& -(1-\beta) I-k^{*}(\operatorname{sgn}(S(t))) .
\end{aligned}
$$

Similar to Theorem 1 and Theorem 2, we can obtain the following result.

Corollary 1. Suppose that the assumptions 1, 2 and 3 hold, systems (7) and (27) are globally projective synchronized based on the controller (29).

when $\beta=1$, the control input (17) is reduced to the following form

$$
\begin{aligned}
u(t)= & u_{e q}+u_{r} \\
= & -(C-D+K P) e(t)-(C-D) x(t)-B g(x(t)) \\
& +A f(x(t))-H+I-k^{*}(\operatorname{sgn}(S(t))),
\end{aligned}
$$

where the sliding surface $S(t)$ is defined in (11). As a special case of Theorem 1 and Theorem 2, the following result is given.

Corollary 2. Under the assumptions 1, 2 and 3, system (7) and system (9) are globally asymptotically complete synchronized based on the controller (30).

When $\beta=-1$, the control input (17) can be rewritten as

$$
\begin{aligned}
u(t)= & u_{e q}+u_{r} \\
= & -(C-D+K P) e(t)+(C-D) x(t)-B g(-x(t)) \\
& -A f(x(t))-H-I-k^{*}(\operatorname{sgn}(S(t))),
\end{aligned}
$$

the sliding surface $S(t)$ is the same as (11). In this case, the following result is deduced directly from Theorem 1 and Theorem 2. 
Corollary 3. Under the assumptions 1, 2 and 3, system (7) and system (9) are globally asymptotically anti-synchronized based on the controller (31).

Remark 5. Since the control signal (17) contains the discontinuous function $\operatorname{sgn}(\cdot)$, the $\operatorname{sgn}(\cdot)$ term may produces some undesirable oscillations known as the chattering. As described in (Aghababa, 2013b; Aghababa \& Khanmohammadi et al., 2011), we replace the $\operatorname{sgn}(\cdot)$ by a continuous function $\tanh (\cdot)$ to attenuate the chattering. Therefore, the control law (17) is modified as follows:

$$
\begin{aligned}
u(t)= & -(C-D+K P) e(t)-\beta(C-D) x(t)-B g(\beta x(t)) \\
& +\beta A f(x(t))-H+\beta I-k^{*}(\tanh (S(t))),
\end{aligned}
$$

Remark 6. In this paper, we discuss the projective synchronization for the different FNNs. Although some scholars studied projective synchronization of FNNs, most results were about the same FNNs (Bao \& Cao, 2015; Yu \& Hu et al., 2014). In fact, the systems are inevitably subject to some environmental changes, which may render their parameters to be variant. In other words, it is hard to keep two systems to be identical all the time. Therefore, our results are necessary and general.

Remark 7. In the existing works, linear feedback controller (Chen \& Zeng et al., 2014; Zhang \& Yu et al., 2015) or adaptive feedback controller (Bao \& Cao, 2015; Yu \& Hu et al., 2014) are often adopted to deal with synchronization problem of FNNs. When consider synchronization problem for two different FNNs, the controllers are not applied because of the difference of form and parameters in this case. Whereas, sliding mode control can overcome this difficulty. Therefore, our results achieve a valuable improvement.

Remark 8. The proposed methods in this paper can be applied to study the projective synchronization of the chaotic and hyperchaotic systems with fractional-order. The fractional order $\alpha$ in projective synchronization for fractional-order chaotic systems is unpredictable so that it can enhance the security of communication additionally. In other word, projective synchronization for FNNs could be used to get more secure communication in application to secure communication comparing with projective synchronization for integer-order systems.

\section{Numerical example}

In this section, one example is provided to verify the effectiveness of results obtained in the previous section.

Example 1: Consider the following three-dimensional FNNs as the drive system

$$
{ }_{0} D_{t}^{\alpha} x(t)=-C x(t)+A f(x(t))+I, \quad t \geq 0,
$$

where $\alpha=0.98, f(x(t))=\tanh (x(t)), I=\left[\begin{array}{lll}0 & 0 & 0\end{array}\right]^{T}$,

$$
C=\left[\begin{array}{lll}
6 & 0 & 0 \\
0 & 6 & 0 \\
0 & 0 & 3
\end{array}\right], A=\left[\begin{array}{ccc}
8 & 5.2 & 6 \\
-1.2 & 2 & 1.15 \\
-4.75 & 0 & 3.1
\end{array}\right] \text {. }
$$

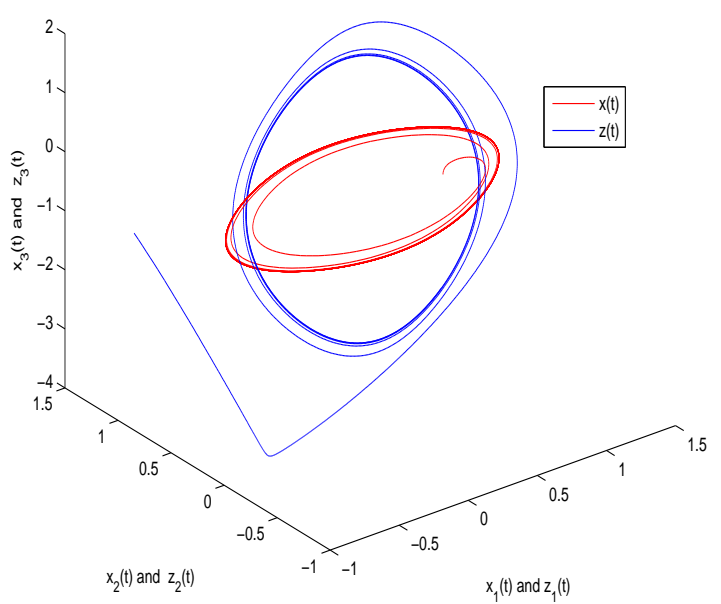

Figure 1: Phase plot of drive system (32) with initial condition $x(0)=$ $(0.2,-0.5,0.8)^{T}$ and phase plot response system (34) with initial values $z(0)=$ $(-0.5,1.5,-1.8)^{T}$ and without the controller $u(t)$.

$$
y(t)=\operatorname{Px}(t),
$$

where the parameter of the measured output (33) is given as $P=\left[\begin{array}{lll}0 & 1 & 0\end{array}\right]$. Then the corresponding response system is as follows:

$$
{ }_{0} D_{t}^{\alpha} z(t)=-D z(t)+B g(z(t))+H+u(t), \quad t \geq 0,
$$

where $\alpha=0.98, g(z(t))=\sin (z(t)), H=\left[\begin{array}{lll}0 & 0 & 0\end{array}\right]^{T}$,

$$
D=\left[\begin{array}{ccc}
0.8 & 0 & 0 \\
0 & 1.2 & 0 \\
0 & 0 & 0.5
\end{array}\right], B=\left[\begin{array}{ccc}
1.5 & -1.2 & -0.5 \\
1.3 & -1.5 & 1.15 \\
2.75 & -2 & 1.15
\end{array}\right]
$$

Fig. 1 depicts the phase trajectories of drive system (32) with initial values $x(0)=(0.2,-0.5,0.8)^{T}$. At the same time, when there is no the controller $u(t)$ in (34), the behaviors of response system (34) with initial values $z(0)=(-0.5,1.5,-1.8)^{T}$ is described.

In control scheme (17), choosing the projective coefficient $\beta=3$ and the gain matrix $K$ can be chosen as $K=[1-$ $\left.\begin{array}{ll}1 & 0.5\end{array}\right]^{T}$. The driver-response systems (32) and (34) are globally asymptotically projective synchronized, which is demonstrated in Figs. 2-3.

In Fig. 2, the projective synchronization errors with different initial values converge to zero, which show that drive system (32) and response system (34) are globally asymptotically projective synchronized with $\beta=3$. And they are verified in Figs. 3 with initial values $x(0)=(0.2,-0.5,0.8)^{T}$, $z(0)=(-0.5,1.5,-1.8)^{T}$.

Similarly, the simulation results for $\beta=-2$ are showed in Figs. 4-5. In this case, we choose the gain matrix $K=[2-$ $\left.\begin{array}{ll}1.5 & 0.5\end{array}\right]^{T}$. 

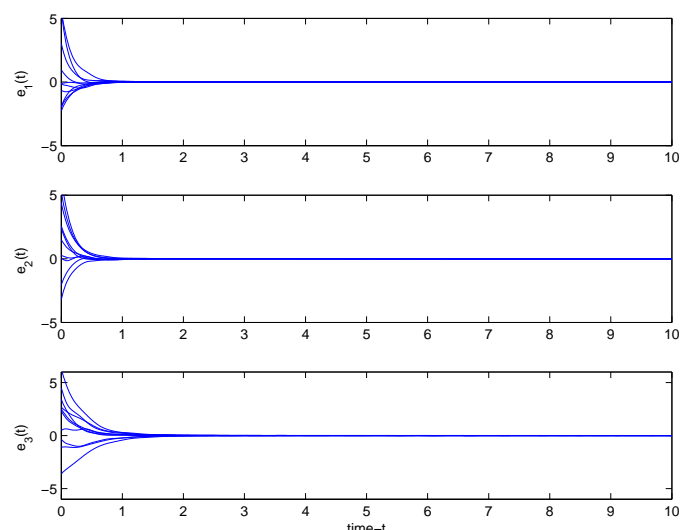

Figure 2: Choose 10 random initial conditions, the synchronization errors $e_{1}(t)$, $e_{2}(t)$ and $e_{3}(t)$ with $\beta=3$.
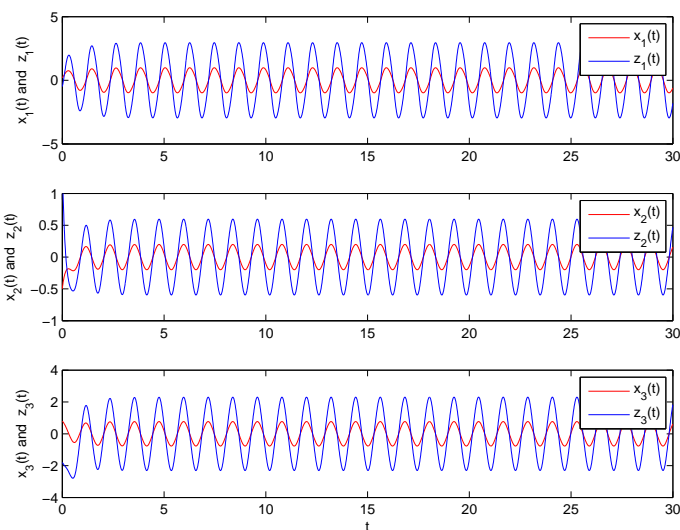

Figure 3: Evolutions of driver-response systems with $\beta=3$.
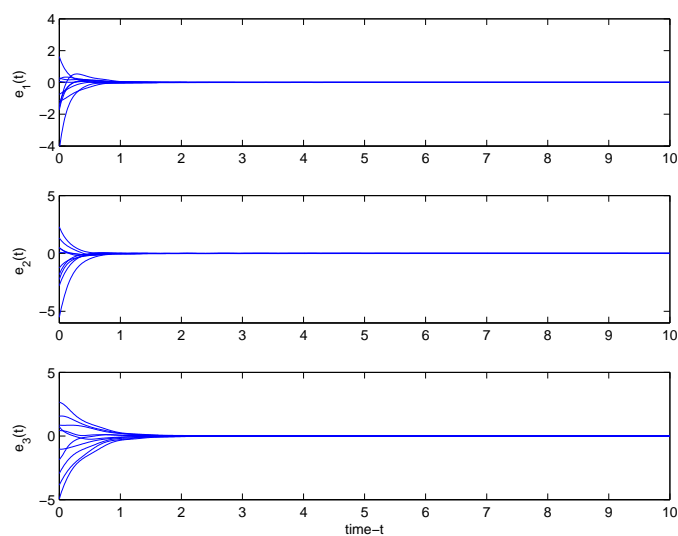

Figure 4: Choose 10 random initial conditions, the synchronization errors $e_{1}(t)$, $e_{2}(t)$ and $e_{3}(t)$ with $\beta=-2$.
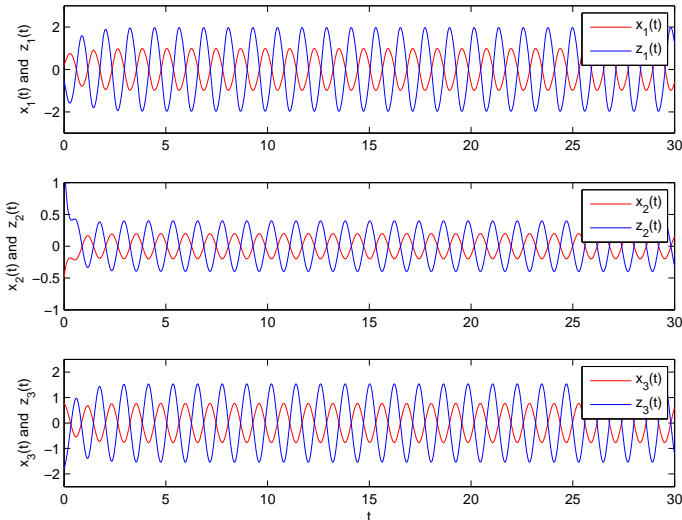

Figure 5: Evolutions of driver-response systems with $\beta=-2$.
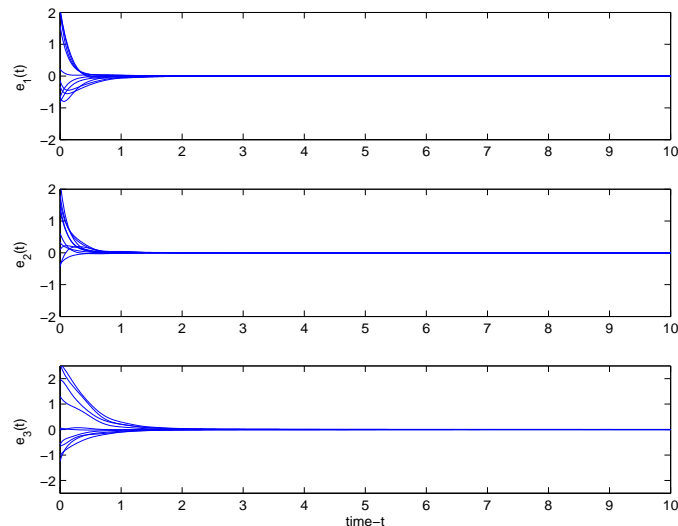

Figure 6: Choose 10 random initial conditions, the synchronization errors $e_{1}(t)$, $e_{2}(t)$ and $e_{3}(t)$ with $\beta=1$.
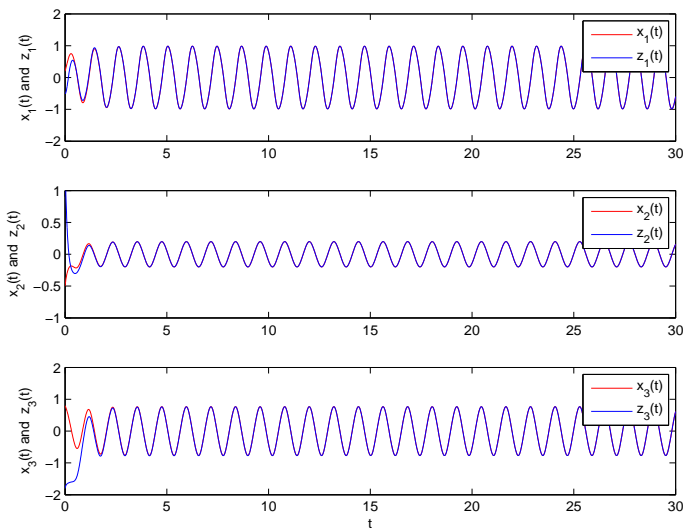

Figure 7: State trajectories of driver-response systems with $\beta=1$. 

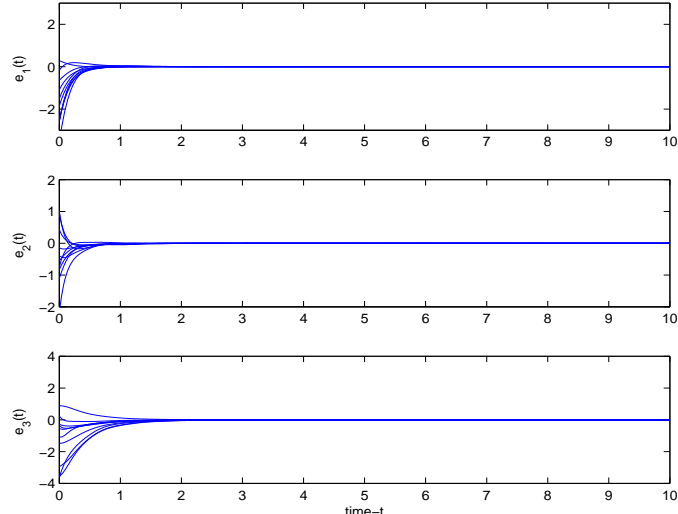

Figure 8: Choose 10 random initial conditions, the synchronization errors $e_{1}(t)$, $e_{2}(t)$ and $e_{3}(t)$ with $\beta=-1$.
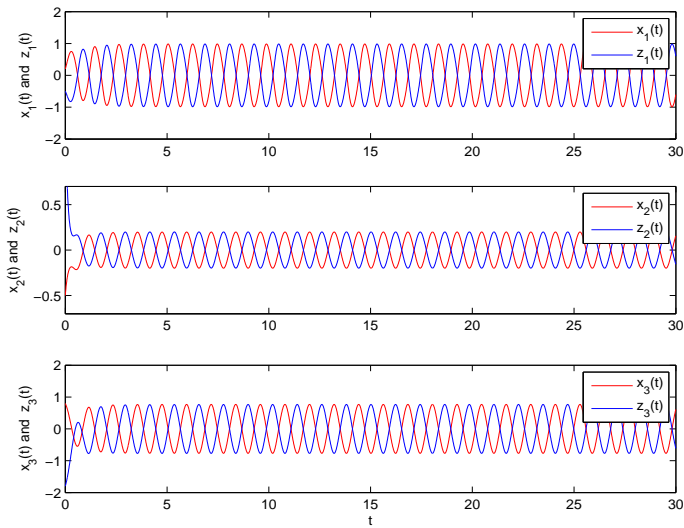

Figure 9: State trajectories of driver-response systems with $\beta=-1$.

In the following, we consider the globally asymptotically complete synchronization of system (32) and system (34). In this case, $\beta=1$ and we choose the gain matrix $K=\left[\begin{array}{lll}2.5 & 0\end{array}\right.$ $1.5]^{T}$. It follows from Corollary 2 that drive system (32) and response system (34) are globally asymptotically complete synchronized. The synchronization errors and synchronization evolutions are presented in Figs. 6-7, respectively.

Similarly, the simulation results for $\beta=-1$ with the gain matrix $K=\left[\begin{array}{lll}0 & 1 & 0\end{array}\right]^{T}$ are showed in Figs. 8-9. Which imply that system (32) and system (34) are globally asymptotically anti-synchronized from Corollary 3.

\section{Conclusions}

In this paper, the global projective synchronization has been investigated for nonidentical FNNs, which is more complex and challenging comparing with projective synchronization of identical FNNs. Some effective criteria are derived based on a fractional-order integral sliding mode controller, sliding mode control theory and fractional Lyapunov direct methods. It is worthwhile to note that the complete synchronization of non- identical FNNs, anti-synchronization of nonidentical FNNs and projective synchronization of identical FNNs are guaranteed as some special cases of our main results. In the future, we will consider the projective synchronization of nonidentical FNNs with delays.

\section{Acknowledgements}

This work was supported by the Key Program of National Natural Science Foundation of China (Grant No. 61134012), the National Science Foundation of China (Grant Nos. 11271146 and 61374150), and the prior developing Field for the Doctoral Program of Higher Education of China (Grant No. 20130142130012).

\section{References}

Aghababa, M. P. (2013). No-chatter variable structure control for fractional nonlinear complex systems. Nonlinear Dynamics, 73, 2329-2342.

Aghababa, M. P. (2013). A novel terminal sliding mode controller for a class of non-autonomous fractional-order systems. Nonlinear Dynamics, 73, 679688.

Aghababa, M. P., Khanmohammadi, S., \& Alizadeh, G. (2011). Finite-time synchronization of two different chaotic systems with unknown parameters via sliding mode technique. Applied Mathematical Modelling, 35, 30803091.

Bao, H. B., \& Cao, J. D. (2015). Projective synchronization of fractional-order memristor-based neural networks. Neural Networks, 63, 1-9.

Boccaletti, S., Kurths, J., Osipov, G., Valladares, D. L., \& Zhou, C. S. (2002). The synchronization of chaotic systems. Physics reports, 366, 1-101.

Carpinteri, A., Cornetti, P., \& Kolwankar, K. M. (2004). Calculation of the tensile and flexural strength of disordered materials using fractional calculus. Chaos, Solitons and Fractals, 21, 623-632.

Chen, G., \& Dong, X. (1998). From chaos to order: methodologies, perspectives and applications. World Scientific, Singapore.

Chen, J., Zeng, Z., \& Jiang, P. (2014). Global Mittag-Leffler stability and synchronization of memristor-based fractional-order neural networks. Neural Networks, 51, 1-8.

Chen, L., Chai, Y., Wu, R., Ma, T., \& Zhai, H. (2013). Dynamic analysis of a class of fractional-order neural networks with delay. Neurocomputing, 111, 190-194.

Chen, W., Ye, L., \& Sun, H. (2010). Fractional diffusion equations by the Kansa method. Computers and Mathematics with Applications, 59, 1614-1620.

Ding, Z., Shen, Y., \& Wang, L. (2016). Global Mittag-Leffler synchronization of fractional-order neural networks with discontinuous activations. Neural Networks, 73, 77-85.

Erjaee, G. H., \& Momani, S. (2008). Phase synchronization in fractional differential chaotic systems. Physics Letters A, 372, 2350-2354.

Gan, Q., Xu, R., \& Kang, X. (2011). Synchronization of chaotic neural networks with mixed time delays. Communications in Nonlinear Science and Numerical Simulation, 16, 966-974.

Hu, M., Xu, Z., \& Yang, Y. (2008). Projective cluster synchronization in driveresponse dynamical networks. Physica A: Statistical Mechanics and its Applications, 387, 3759-3768.

Huang, H., \& Feng, G. (2009). Synchronization of nonidentical chaotic neural networks with time delays. Neural networks, 22, 869-874.

Isfer, L. A. D., Lenzi, E. K., Teixeira, G. M., \& Lenzi, M. K. (2010). Fractional control of an industrial furnace. Acta Scientiarum. Technology, 32, 279-285.

Kaslik, E., \& Sivasundaram, S. (2012). Nonlinear dynamics and chaos in fractional-order neural networks. Neural Networks, 32, 245-256.

Kilbas, A. A., \& Marzan, S. A. (2005). Nonlinear differential equations with the Caputo fractional derivative in the space of continuously differentiable functions. Differential Equations, 41, 84-89.

Kilbas, A. A. A., Srivastava, H. M., \& Trujillo, J. J. (2006). Theory and applications of fractional differential equations. Elsevier.

Li, C. P., \& Deng, W. H. (2007). Remarks on fractional derivatives. Applied Mathematics and Computation, 187, 777-784. 
Li, Y., Chen, Y., \& Podlubny, I. (2009). Mittag-Leffler stability of fractional order nonlinear dynamic systems. Automatica, 45, 1965-1969.

Li, Y., Chen, Y., \& Podlubny, I. (2010). Stability of fractional-order nonlinear dynamic systems: Lyapunov direct method and generalized Mittag-Leffler stability. Computers and Mathematics with Applications, 59, 1810-1821.

Magin, R. L. (2004). Fractional calculus in bioengineering, part 3. Critical Reviews in Biomedical Engineering, 32, 195-377.

Magin, R. L. (2010). Fractional calculus models of complex dynamics in biological tissues. Computers and Mathematics with Applications, 59, 15861593.

Magin, R. L., \& Ovadia, M. (2008). Modeling the cardiac tissue electrode interface using fractional calculus. Journal of Vibration and Control, 14, 14311442.

Ojalvo, J. G., \& Roy, R. (2001). Spatiotemporal Communication with Synchronized Optical Chaos. Physical Review Letters, 86, 5204-5207.

Ott, E., Grebogi, C., \& Yorke, J. A. (1990). Controlling chaos. Physical review letters, 64, 1196-1199.

Pecora, L. M., \& Carroll, T. L. (1990). Synchronization in chaotic system. Physical Review Letters, 64, 821-824.

Podlubny, I. (1999). Fractional differential equations. San Diego, California: Academic Press.

Si, G. Q., Sun, Z. Y., Zhang, Y. B., \& Chen, W. Q. (2012). Projective synchronization of different fractional-order chaotic systems with non-identical orders. Nomlinear Analysis: Real World Applications, 13, 1761-1771.

Slotine, J. J. E., \& Li, W. (1991). Applied nonlinear control. Englewood Cliffs, NJ: Prentice-hall.

Soczkiewicz, E. (2002). Application of fractional calculus in the theory of viscoelasticity. Molecular and Quantum Acoustics, 23, 397-404.

Sprott, J. C. (2003). Chaos and time-series analysis. Oxford: Oxford University Press.

Szabo, T. L., \& Wu, J. (2000). A model for longitudinal and shear wave propagation in viscoelastic media. The Journal of the Acoustical Society of America, 107, 2437-2446.

Tripathi, D., Pandey, S. K., \& Das, S. (2010). Peristaltic flow of viscoelastic fluid with fractional Maxwell model through a channel. Applied Mathematics and Computation, 215, 3645-3654.
Wang, H., Yu, Y., Wen, G., \& Zhang, S. (2013). Stability analysis of fractionalorder neural networks with time delay. Neural Processing Letters, 1-22.

Wang L., Shen, Y., Yin, Q., \& Zhang, G. (2015). Adaptive synchronization of memristor-based neural networks with time-varying delays. IEEE Transactions on Neural Networks and Learning Systems, 26, 2033-2042.

Wang, L., Shen, Y., \& Ding, Z. (2015). Finite time stabilization of delayed neural networks. Nueral Networks, 70, 74-80.

Wang, X., \& He, Y. (2008). Projective synchronization of fractional order chaotic system based on linear separation. Physics Letters A, 372, 435-441.

Wu, X. J., Lai, D. R., \& Lu, H. T. (2012). Generalized synchronization of fractional-order chaos in weighted complex dynamical networks with nonidential nodes. Nonlinear Dynamics, 69, 667-683.

Xin, B. G., Chen, T., \& Liu, Y. Q. (2011). Projective synchronization of chaotic fractional-order energy resources demand-supply systems via linear control. Commun Nonlinear Sci Numer Simulat., 16, 4479-4486.

Yan, J., \& Li, C. (2007). On chaos synchronization of fractional differential equations. Chaos, Solitons and Fractals, 32, 725-735.

Yang, T., \& Chua, L. O. (1997). Impulsive stabilization for control and synchronization of chaotic systems: theory and application to secure communication. Circuits and Systems I: Fundamental Theory and Applications, IEEE Transactions on, 44, 976-988.

Yu, J., Hu, C., \& Jiang, H. (2012). $\alpha$-stability and $\alpha$-synchronization for fractional-order neural networks. Neural Networks, 35, 82-87.

Yu, J., Hu, C., Jiang, H., \& Fan, X. (2014). Projective synchronization for fractional neural networks. Neural Networks, 49, 87-95.

Zhang, S., Yu, Y., \& Wang, H. (2015). Mittag-Leffler stability of fractionalorder Hopfield neural networks. Nonlinear Analysis: Hybrid Systems, 16, 104-121.

Zhou, S., Li, H., \& Zhu, Z. (2008). Chaos control and synchronization in a fractional neuron network system. Chaos, Solitons and Fractals, 36, 973 984.

Zhu, H., He, Z., \& Zhou, S. (2011). Lag Synchronization of the FractionalOrder System via Nonlinear Observer. International Journal of Modern Physics B, 25, 3951-3964.

Zou, T., Qu, J., Chen, L., Chai, Y., \& Yang, Z. (2014). Stability analysis of a class of fractional-order neural networks. TELKOMNIKA Indonesian Journal of Electrical Engineering, 12, 1086-1093. 